\title{
The very forward hadron calorimeter PSD for the future CBM@FAIR experiment
}

\author{
$V$. Mikhaylov ${ }^{1,2, *}, A$. Kugler ${ }^{1}, V$. Kushpil ${ }^{1}, O$. Svoboda $^{1}, P$. Tlustý ${ }^{1}, M$. Golubeva $^{3}, F$. Guber $^{3}$,

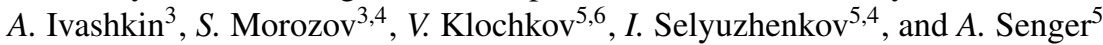 \\ for the CBM Collaboration \\ ${ }^{1}$ Nuclear Physics Institute of CAS, Řež, Czech Republic \\ ${ }^{2}$ Czech Technical University in Prague, Czech Republic \\ ${ }^{3}$ Institute for Nuclear Research RAS, Moscow, Russia \\ ${ }^{4}$ National Research Nuclear University MEPhI, Moscow, Russia \\ ${ }^{5}$ GSI Helmholtz Centre for Heavy Ion Research, Darmstadt, Germany \\ ${ }^{6}$ Goethe University Frankfurt, Frankfurt am Main, Germany
}

\begin{abstract}
The Projectile Spectator Detector (PSD) of the CBM experiment at the future FAIR facility is a compensating lead-scintillator calorimeter designed to measure the energy distribution of the forward going projectile nucleons and nuclei fragments (reaction spectators) produced close to the beam rapidity. The detector performance for the centrality and reaction plane determination is reviewed based on Monte-Carlo simulations of gold-gold collisions by means of four different heavy-ion event generators. The PSD energy resolution and the linearity of the response measured at CERN PS for the PSD supermodule consisting of 9 modules are presented. Predictions of the calorimeter radiation conditions at CBM and response measurement of one PSD module equipped with neutron irradiated MPPCs used for the light read out are discussed.
\end{abstract}

\section{Introduction}

The Compressed Baryonic Matter (CBM) experiment at the future Facility for Antiproton and Ion Research (FAIR) will investigate the region of the QCD phase diagram of high net-baryon densities studying heavy-ion collisions. The PSD calorimeter will provide information on the centrality and reaction plane orientation of the nucleus-nucleus collisions at the SIS-100 beam energy range of $2-10 \mathrm{AGeV}$ with beam interaction rates up to $1 \mathrm{MHz}$ [1]. PSD has a modular transverse structure consisting of 44 modules with a beam hole in the center of the calorimeter, see Fig. 1 (left). The design of a support platform will allow the detector movement along all 3 axes [2]. Each detector module has 5.6 hadron interaction lengths and transverse size of $20 \times 20 \mathrm{~cm}^{2}$. Fig. 1 (right) depicts the module structure consisting of 60 lead/scintillator sandwiches with the sampling ratio of $4: 1$. Light from each of 6 consecutive scintillator plates in the module is collected by WLS fibers and is read out by one photodetector, $3 \times 3 \mathrm{~mm}^{2}$ Hamamatsu S12572-010P MPPC placed at the end of the module. Thus, the module has the readout from 10 longitudinal sections that allows to measure the longitudinal hadron shower profile and ensure the uniformity of light collection along the module.

\footnotetext{
*e-mail: mikhaylov@ujf.cas.cz
} 
Fig. 1 (center) introduces the PSD supermodule consisting of 9 modules assembled in $3 \times 3$ array at CERN to study the calorimeter performance.
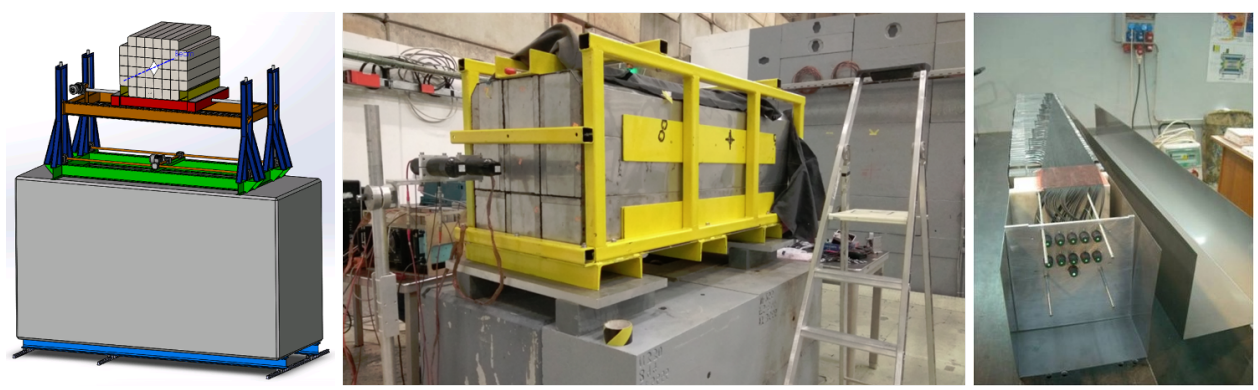

Figure 1. (Left) Design of PSD with a support platform [2]. (Center) PSD supermodule. (Right) PSD module during assembly

\section{PSD performance simulations}

To study the detector performance, the $\mathrm{Au}+\mathrm{Au}$ collision events in the energy range from 1 to $30 \mathrm{AGeV}$ were generated by means of UrQMD, DCM-QGSM, LA-QGSM and HSD heavy-ion models [3]. CBMROOT was used to simulate the detector response to particles transported with GEANT4 through the CBM setup. Utilized models exhibit variation in the directed particle flow $v_{1}$ which describes the asymmetry in the azimuthal distributions of produced particles with respect to the initial reaction plane [3]. The substantial difference in $v_{1}$ strength is observed for the midrapidity region of $-0.5<y_{\text {norm }}<0.5$ as presented in Fig. 2 (left). However, Fig. 2 (right) shows much smaller difference in $v_{1}$ around the beam rapidity $y_{\text {norm }} \sim 1$.
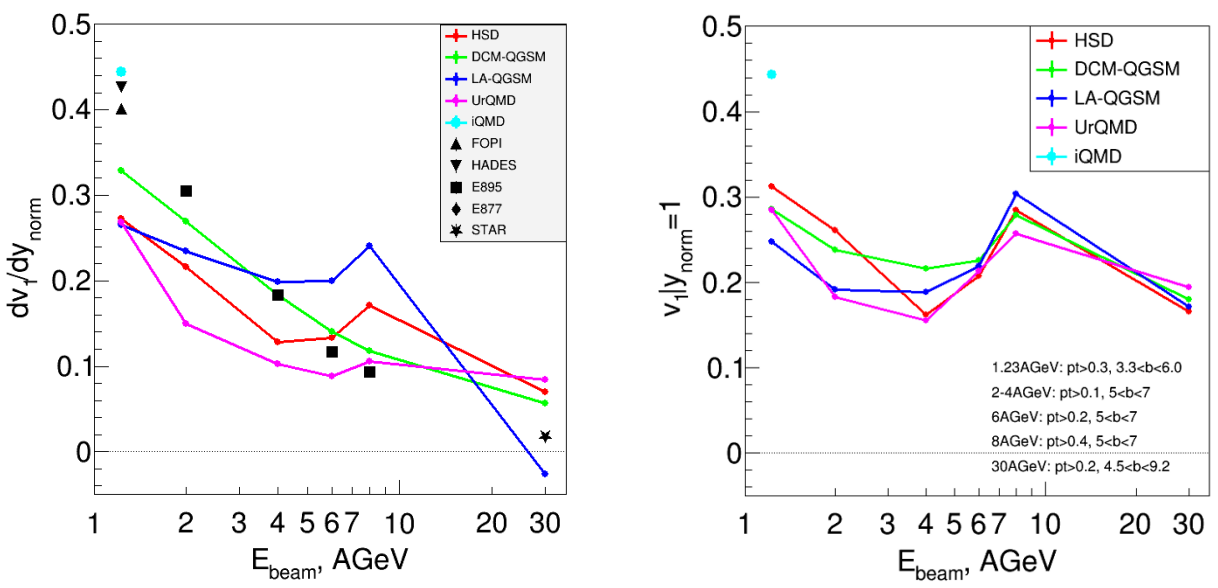

Figure 2. Integrated particle flow vs beam energy around midrapidity (left) and projectile rapidity (right). Simulations are done by means of different collision models for proton directed flow at $\mathrm{Au}+\mathrm{Au}$ collisions at semi-centrality with $b$ and $p_{\mathrm{T}}$ cuts corresponding to indicated experimental data 
Fig. 3 (left) shows the performance of PSD reaction plane resolution simulated with input from four event generators. The reaction plane resolution is defined as a Gaussian standard deviation of the $\Psi_{\mathrm{EP}}-\Psi_{\mathrm{RP}}$ distribution, where $\Psi_{\mathrm{RP}}$ is the true reaction plane extracted from the model data and $\Psi_{\mathrm{EP}}$ is an event plane angle estimated by the anisotropy of energy deposition in PSD modules. Therefore, strength of $v_{1}$ determines precision of the reaction plane reconstruction. The $\sigma\left(\Psi_{\mathrm{EP}}-\Psi_{\mathrm{RP}}\right)$ resolution is below 40 degrees for beam energies higher than $4 \mathrm{AGeV}$ for all the utilized models. This is consistent with the observed small variation in $v_{1}$ around the beam rapidity where PSD detects spectators. Availability of nuclei fragments only in DCM-QGSM and LA-QGSM generators also contributes to the difference in the resolution.
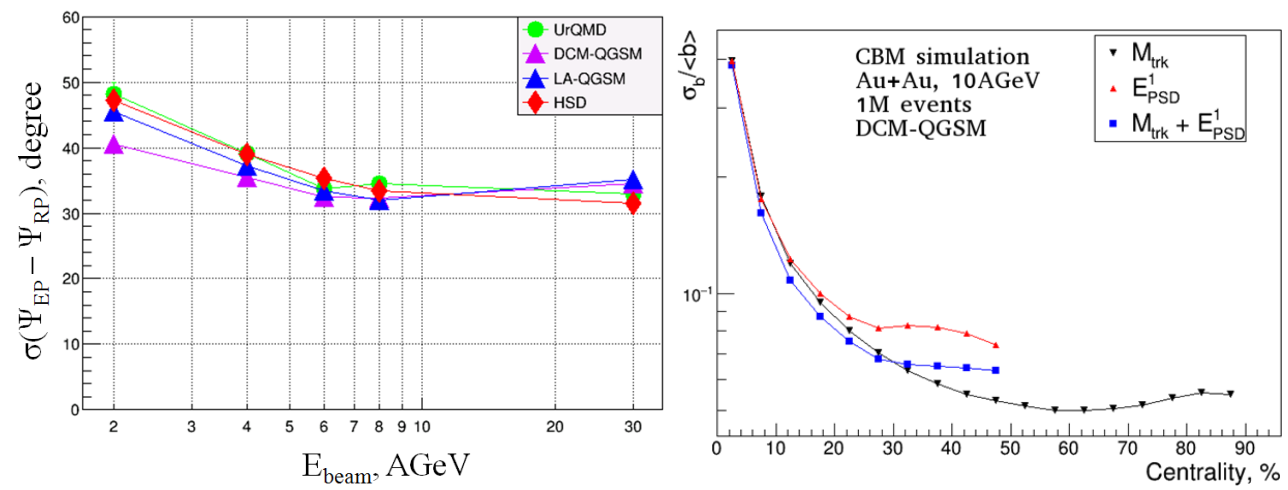

Figure 3. (Left) Reaction plane resolution vs beam energy for various collision models, 100K events were generated for every energy and every model. (Right) Impact parameter resolution for different centrality estimators, $1 \mathrm{M}$ events of $10 \mathrm{AGeV} \mathrm{Au+Au}$ collisions were generated with DCM-QGSM model

Fig. 3 (right) presents the resolution of impact parameter $b$ defined as a ratio of the $b$ distribution spread $\sigma_{b}$ to its average $\langle b\rangle$ for a given centrality. All events are sorted in centrality classes, with most central $(b \approx 0$ or centrality $\approx 0 \%)$ being the collisions with the highest multiplicity of the produced particles and peripheral $(b \approx 2$ nuclear radii or centrality $\approx 100 \%$ ) with low multiplicity. Resolutions of $b$ are compared for estimators based on different CBM subsystems. Charged particle tracks multiplicity $M_{t r k}$ was reconstructed with STS and MVD detectors which cover the polar range where the reaction participants are emitted. Energy of spectators was reconstructed with inner four modules of PSD combined into PSD subgroup. Resulting impact parameter resolution is about $5-10 \%$ for mid-central and peripheral events. PSD $b$ resolution is comparable to that of STS+MVD for centrality up to $30 \%$, i.e. for central and semi-peripheral collisions. In peripheral events there are fragments which go into the beam hole and therefore cannot be detected in the PSD limiting PSD centrality determination to $0-50 \%$. Nevertheless, independent centrality determination in regions of reaction participants and spectators is very important, for instance for the fluctuations study, etc. The detailed information on centrality determination in CBM can be found at [4].

\section{PSD supermodule performance at CERN PS}

Supermodule response was studied with proton beams in the momentum range of $2-10$ $\mathrm{GeV} / \mathrm{c}$ at the CERN Proton Synchrotron (PS) T9 and T10 beam lines. For the first time, the 

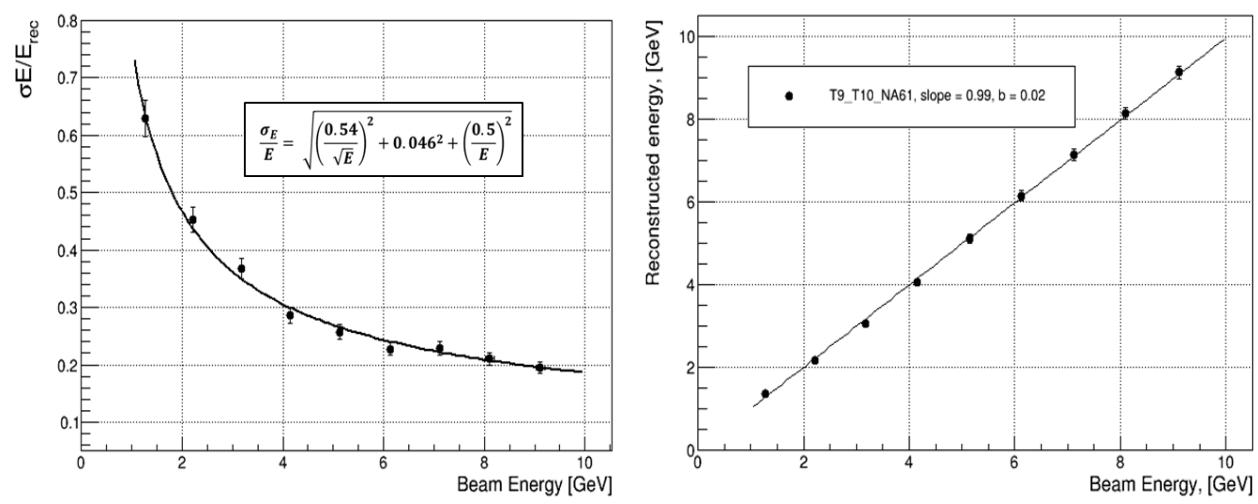

Figure 4. Supermodule energy resolution (left) and linearity (right) vs proton beam energy

energy resolution and the linearity of the response have been measured at these low energies. Detector calibration was done by means of beam muons acting as MIP particles which produced the light yield of about 8-10 photoelectrons/MeV in each section. Protons were identified by TOF method using two quartz Cherenkov detectors installed in front of the supermodule at a distance of about $12 \mathrm{~m}$ from each other with a time resolution of about 60 psec. Fig. 4 demonstrates the measured supermodule relative energy resolution (left) and linearity (right). The energy resolution $\sigma_{E} / E$ is estimated to be about $54 \% / \sqrt{E(\mathrm{GeV})}$ along with the deflection from linearity less than $1 \%$ which satisfies the requirements of the CBM experiment [1].

\section{PSD performance under the radiation conditions}

The calorimeter irradiation was simulated with the FLUKA code for 2 months of the CBM run with $\mathrm{Au}+\mathrm{Au}$ collisions on a $1 \%$ interaction $\mathrm{Au}$ target at the beam energy of $10 \mathrm{AGeV}$ and beam rate of $10^{8}$ ions/s. Fig. 5 (left) shows that the absorbed dose in the PSD does not exceed $1 \mathrm{kGy}$. Damage by the ionizing dose which is the most crucial for the scintillator tiles was studied in details for calorimeters of $\mathrm{LHCb}$ experiment [5]. The relative light yield of the LHCb tiles degraded by $25 \%$ after $2.5 \mathrm{kGy}$ irradiation, and then slowly decreased further by $20 \%$ after irradiation up to $14 \mathrm{kGy}$. Therefore, scintillators and WLS-fibers of PSD are not expected to degrade significantly. Moreover, the modular structure and the longitudinal segmentation of PSD coupled with the regular calibration will ensure the stability of transverse and longitudinal uniformity of the light collection.

To avoid a high radiation load in the central modules, there is a beam hole in the center of PSD. Three different beam hole shapes were considered, namely, round with a $3 \mathrm{~cm}$ radius, quadratic- and diamond-shape of $20 \times 20 \mathrm{~cm}^{2}$ size. Fig. 5 (right) illustrates the reduction of non-ionizing energy loss by a factor of 2 at the distance of $10 \mathrm{~cm}$ from the beam axis for the enlarged beam hole. Diamond-shaped hole design was chosen because it provided additional reduction of the ionizing dose compared to the quadratic-shaped design [6]. Another safety measure in form of a dedicated neutron shielding based on $8 \mathrm{~cm}$ thick polyethylene blocks with $3 \%$ of boron is introduced reducing the fluence up to factor of five.

Non-ionizing energy losses are important for semiconductor readout electronics and most crucial for the Silicon Photomultipliers or Multi-Pixel Photon Counters (MPPCs) which have a fine pixel structure of $10 \times 10 \mu \mathrm{m}^{2} .1 \mathrm{MeV}$ equivalent neutron fluence at the very end of 

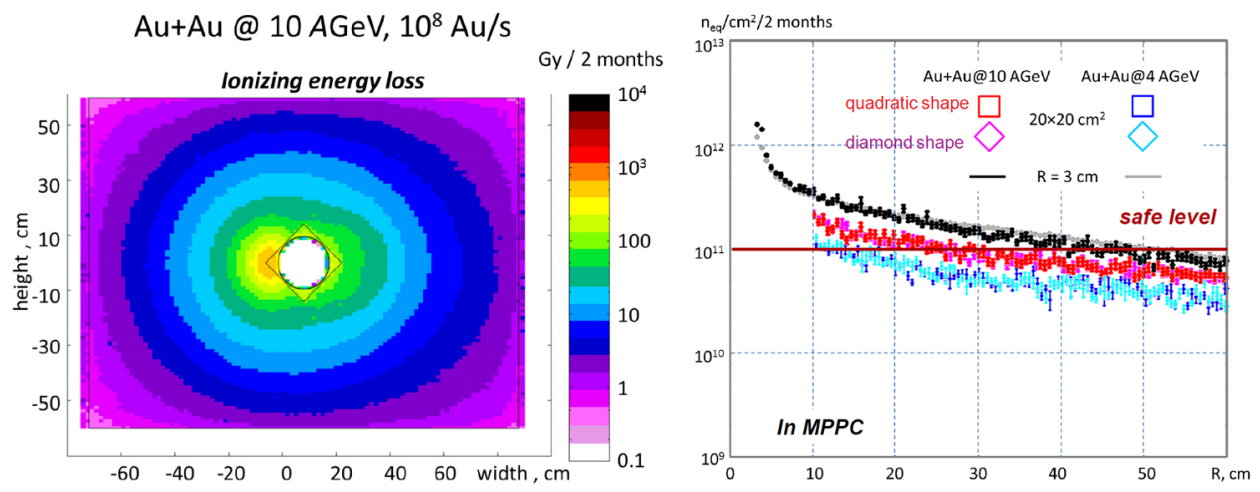

Figure 5. (Left) Distribution of ionizing energy loss in transverse plane at $20 \mathrm{~cm}$ depth from the PSD module front. (Right) Non-ionizing energy loss vs radius at transverse plane where MPPCs are located for different configurations of the beam hole

the calorimeter where the MPPCs are located is below $2 \times 10^{11} \mathrm{n}_{e q} / \mathrm{cm}^{2}$ for inner calorimeter modules. To study the effect of neutron fluence on MPPCs, several samples were irradiated at the cyclotron of NPI with a broad (from thermal up to $34 \mathrm{MeV}$ [7]) neutron spectrum and total fluences in the wide range of $6 \times 10^{10}-9 \times 10^{12} \mathrm{n}_{e q} / \mathrm{cm}^{2}$. After irradiation with $2 \times 10^{11} \mathrm{n}_{e q} / \mathrm{cm}^{2}$ MPPC the dark current increased by 3 orders of magnitude and reached 10 $\mu \mathrm{A}$ [8]. This resulted in the increase of noise by 1 order of magnitude and consequent drop of the signal to noise ratio down to about 50 .
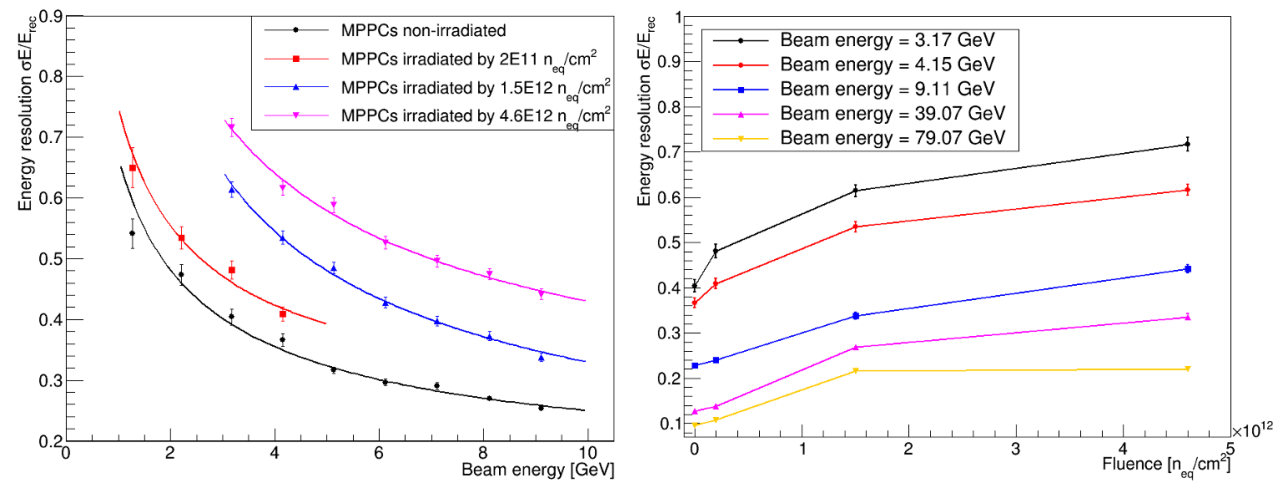

Figure 6. Single module proton energy resolution vs beam energies for different neutron fluences acquired by MPPCs (left) and vs fluence for different beam energies (right)

To study the calorimeter performance degradation due to the MPPC irradiation, single PSD module was consequently equipped with 3 batches of irradiated photodiodes. The neutron fluences accumulated by MPPCs were $2 \times 10^{11}, 1.5 \times 10^{12}$ and $4.6 \times 10^{12} \mathrm{n}_{e q} / \mathrm{cm}^{2}$. Single module response to the proton beams measured inside the PSD supermodule in the momentum range of $2-10 \mathrm{GeV} / \mathrm{c}$ is shown in Fig. 6 (left). Another test was performed with beams of up to $80 \mathrm{GeV} / \mathrm{c}$ momentum at calorimeter of NA61 experiment in CERN which has a sim- 
ilar module design as CBM PSD [9]. Detailed information on this test can be found at [8] ${ }^{1}$. Summary of the energy resolution degradation for both tests is presented in Fig. 6 (right). PSD module energy resolution drops only slightly after irradiation by $2 \times 10^{11} \mathrm{n}_{e q} / \mathrm{cm}^{2}$. Furthermore, the inner calorimeter modules are located closer to the beam and typically detect higher signals which enhance signal to noise ratio and energy resolution. On the other hand, the modules located further from the beam will achieve a significantly lower dose which again improves energy resolution.

\section{Conclusion}

The overview of the recent investigations on the CBM PSD is presented. Detector performance simulations suggest the use of PSD to determine the main collision event characteristics. The impact parameter resolution for the centrality estimation is about $5-10 \%$ and the reaction plane resolution is below 40 degrees. Minor differences in the reaction plane resolution are observed for different heavy-ion collision models explained by the variation in the directed particle flow $v_{1}$ strength in the models.

The PSD supermodule performance test at CERN PS showed the energy resolution about $54 \% / \sqrt{E(\mathrm{GeV})}$ and linearity deflection less than $1 \%$. Due to the harsh radiation conditions a $20 \times 20 \mathrm{~cm}^{2}$ beam hole and endcap neutron shielding are incorporated in design reducing the radiation load by a factor of 10 . The estimated absorbed ionizing dose below $1 \mathrm{kGy}$ is considered to be safe for the operation. High neutron fluence of $2 \times 10^{11} \mathrm{n}_{e q} / \mathrm{cm}^{2}$ results in slight deterioration of energy resolution due to degradation of the MPPC photodiodes.

The achieved results are as well of interest for MPD and BM@N experiments at NICA and NA61 experiment at CERN where a very similar calorimeter design including the MPPCs light readout is used.

Acknowledgements. The authors thank for the help with heavy-ion collisions simulations: Y. Leifels (iQMD), V. Friese (UrQMD), M. Golubeva (DCM-QGSM), K. Gudima (LA-QGSM) and E. Bratkovskaya (HSD). The authors thank the CBM and NA61 collaborations for their support in the tests and thank CERN staff M.Jeckel and L.Gatignon for their help in test preparation at the beamlines. The authors thank the NPI cyclotron and neutron generators team, especially M. Štefánik and M. Majerle, for excellent beam conditions and help with irradiation tests that were carried out at the CANAM infrastructure. This work was supported by Czech MEYS project no. LM2015049 and EU OP VVV CZ.02.1.01/0.0/0.0/16_013/0001677 grant.

\section{References}

[1] F. Guber, I. Selyuzhenkov, eds., Technical Design Report for the CBM Projectile Spectator Detector (PSD) (GSI, Darmstadt, 2015). http://repository.gsi.de/record/109059

[2] M. Janda et al. (2018), presentations at 31st and 32nd CBM Collaboration Meetings, martin.janda(at)fs.cvut.cz

[3] V. Mikhaylov et al., PoS EPS-HEP 2015, 208 (2015)

[4] V. Klochkov, I. Selyuzhenkov (CBM Collaboration), Journal of Physics: Conference Series 798, 012059 (2017)

\footnotetext{
${ }^{1}$ It is important to note, that only first 5 sections of the module were measured for MPPCs irradiated by $1.5 \times 10^{12}$ and $4.6 \times 10^{12} \mathrm{n}_{e q} / \mathrm{cm}^{2}$. Dark currents of these photodiodes increased up to a few $\mathrm{mA}$ after irradiation, thus external voltage supply had to be used which introduced additional noise. Therefore, achieved performance for these highly irradiated MPPCs will be regarded as the upper limit and expected to be much better in reality if these doses will ever be reached in the experiment.
} 
[5] J. Alves, A. Augusto et al. (LHCb Collaboration), JINST 3, S08005 (2008)

[6] A. Senger (2017 - 2018), presentations at 29th - 32nd CBM Collaboration Meetings, A.Senger(at)gsi.de

[7] M. Stefanik, P. Bem, M. Gotz, K. Katovsky, M. Majerle, J. Novak, E. Simeckova, Radiation Physics and Chemistry 104, 306 (2014)

[8] V. Mikhaylov et al., Nucl.Instrum.Meth. A 912, 241-244 (2018)

[9] M. Golubeva et al., J. Phys. Conf. Ser. 798, 012073 (2017) 